Editorial

\title{
ROL SOCIOPOLÍTICO DE ENFERMERAS Y ENFERMEROS VINCULADO CON EL LIDERAZGO Y LA AUTONOMÍA PROFESIONAL
}

\section{SOCIO-POLITICAL ROLE OF NURSES LINKED TO LEADERSHIP AND PROFESSIONAL AUTONOMY}

\section{Cecilia Landman Navarro}

Enfermera-matrona, licenciada en Enfermería

Magíster en Educación m/curriculum, Doctora en Educación m/evaluación educacional

Universidad San Sebastián, Escuela de Enfermería, Santiago, Chile

cecilia.landman@uss.cl

http://dx.doi.org/10.4067/S0717-95532015000100009

\section{http://dx.doi.org/10.7764/Horiz_Enferm.32.2.102-107}

«Nadie puede ser feliz sin participar en la felicidad pública, nadie puede ser libre sin la experiencia de la libertad pública, y nadie, finalmente, puede ser feliz o libre sin implicarse y formar parte del poder político»

Hannah Arendt

\section{INTRODUCCIÓN}

Es tiempo de considerar las oportunidades que actualmente se presentan a enfermeras/os, a raíz de contar con el respaldo legal que define el campo del ejercicio profesional ${ }^{(1-2)}$; disponer de un corpus deontológico consolidado en principios y valores, así como, una definición de las competencias para la gestión del cuidado, para cumplir a cabalidad el compromiso de la enfermería con la sociedad, desde una práctica ética y autónoma para tomar decisiones respecto al cuidado ${ }^{(3)}$.

En los lineamientos del Consejo Internacional de Enfermeras (CIE), se propone que para el desarrollo del rol sociopolítico, a través de funciones esenciales, que junto con los cuidados autónomos y de colaboración, se agreguen la defensa y el fomento de un entorno seguro, la gestión del cuidado y de los sistemas de salud, el desarrollo de investigaciones, la participación en la formación y en las políticas sanitarias ${ }^{(4)}$.

Las/los enfermeras/os muestran diversos niveles de autonomía, se observa una mayor independencia en las funciones derivadas de la asistencia, especialmente en lo referente a educación, promoción en salud y en la realización de procedimientos técnicos, en contrapartida con un menor grado de independencia en las funciones derivadas de la gestión e investigación. Esta situación se ve agravada por la escasa participación de los profesionales en las organizaciones gremiales, lo cual limita una defensa colectiva para el reconocimiento social del rol integrado ${ }^{(5)}$.

Correspondencia email: cecilia.landman@uss.cl 
Para potenciar el cumplimiento de manera integral de las funciones, es necesario fortalecer desde la formación de enfermeras/os la sensibilidad social, el liderazgo, el compromiso político y una comunicación horizontal, a través de un paradigma transformador, que se proyecte más allá de ser un apoyo a la comunidad a resolver sus problemas socio-sanitarios, sino que además, brindarles las herramientas necesarias para el empoderamiento social, a través de un auténtico diálogo social que les permita tomar sus propias decisiones y que se reconozcan como ciudadanos responsables del cumplimiento de sus deberes, conscientes de sus propios derechos en el área sociosanitaria ${ }^{(6)}$.

La enfermería desde sus inicios se ha movilizado en escenarios duales de invisibilización-reconocimiento, sumisión-autonomía, enfermería vocacional femeninaenfermería profesional científica, situaciones que, a su vez, generan un significado paradojal para el colectivo, insatisfacción-enriquecimiento del rol profesional.

\section{DESARROLLANDO LA AUTONOMÍA Y EL LIDERAZGO EN ENFERMERÍA}

La autonomía y el liderazgo, considerados como una entidad diádica en el área de la gestión y administración, que se potencian entre sí, en una amalgama simbólica, para lograr el necesario reconocimiento de los profesionales de enfermería, en un ejercicio de liderazgo participativo, basado en la confianza e interdependencia entre los miembros del equipo de salud, con lo cual se logra, un mayor grado de satisfacción y de compromiso con la institución y del trabajo desempeñado(7).

Diversas investigaciones consideran a la autonomía, como uno de los atributos e indicadores que definen al profesionalismo de la/del enfermera/o, que permite el propio posicionamiento dentro del equipo de salud y condiciona el empoderamiento en el ejercicio de su rol, en cuanto a manifestar independencia en la práctica, como en la aplicación del juicio crítico para tomar decisiones. Implica correr riesgos, responsabilizarse de los propios actos, capacidad de autodeterminación y de autorregulación ${ }^{(8-10)}$.

Al liderazgo se lo relaciona directamente con el ejercicio autónomo de la profesión, entendido como un fenómeno colectivo que implica un proceso de influencia de una persona sobre los demás, con el fin de lograr metas comunes, tanto institucionales como de gestión del cuidado con énfasis en la eficacia y en la entrega de un servicio profesional de alta calidad $^{(11)}$. Peter Druker agrega que el liderazgo efectivo surge del compromiso de servir a los demás, en vez de a uno mismo, potenciando de manera colaborativa a las organizaciones en las cuales se labora ${ }^{(12)}$.

Es importante señalar que las/los enfermeras/os requieren unir sus esfuerzos, en todos los niveles y manifestarse públicamente para lograr su plena visibilidad y garantizar su reconocimiento en el escenario político a través del discurso y acciones consistentes con los requerimientos sociales y de salud de las personas, familias y comunidades.

El progreso profesional y disciplinar de la enfermería ha sido una ruta escarpada, aún persisten escollos que dificultan el liderazgo para asumir en propiedad un rol sociopolítico comprometido con la sociedad y el colectivo. 


\section{AVANZANDO DESDE LA SUBORDINACIÓN A LA AUTONOMÍA}

Se ha realizado un largo camino desde los cuidados domésticos, vocacionales, técnicos, hasta alcanzar la impronta profesional. Sin embargo, se debiera avanzar hacia el profesionalismo, como una apuesta de integración teórica y práctica, que combine el conocimiento, con el saber hacer y el saber moral, de manera tal, que se facilite el aprendizaje para lograr la autonomía decisional en la acción del cuidar y no sólo cumplir con las indicaciones médicas e intervenciones técnicas, esto es, asumir el liderazgo para la gestión del cuidado en su integralidad ${ }^{(13,14)}$.

Podríamos decir que, el espíritu de la subordinación tradicional de las enfermeras y enfermeros al modelo hegemónico biomédico, se basa en el concepto de "facultativo" esencialmente establecido para la profesión médica, esperando que hoy día esté modelo sea realmente superado. El hecho que, en la mayoría de las instituciones formadoras, las carreras de enfermería estén integradas a una facultad de medicina, de alguna manera, esa dependencia impide una genuina autonomía identitaria. Esta es aún, una situación no resuelta, sin embargo, se reconocen algunas señales de retirada, en especial en universidades que valoran el propio desarrollo de la enfermería.

Si bien es cierto, en este último tiempo se ha progresado para el logro de la autonomía y el ejercicio del liderazgo en enfermería, aún no se ha llegado a destino, debido a la persistencia de barreras que se originan por la coexistencia de modelos axiológicos institucionales biologisista y economicista, junto con la humanización del cuidado, dejando al profesional en posiciones de subordinación que desdibujan la imagen del profesional y lo ubican, muchas veces, en posiciones alejadas de los genuinos requerimientos de pacientes, familias y comunidades ${ }^{(5,13,15,16)}$

En esta dualidad del ejercicio del liderazgo y autonomía, corresponde destacar la visibilización de enfermeras/os producto de la actual situación sanitaria, demostrando una capacidad de liderazgo en los servicios de enfermería, tanto en el contexto comunitario, para el testeo, trazabilidad, seguimiento e inmunizaciones, contribuyendo al control de la pandemia; como en la atención cerrada, gestionando y brindando cuidados humanizados e integrales de alta especialización.

Se requiere intencionar una nueva enfermería que asuma como papel principal la visibilización del profesional y mejorar la imagen social proyectada, rompiendo los estereotipos de subordinación ${ }^{(17)}$.

\section{ROL SOCIAL Y POLÍTICO DEL PROFESIONAL DE ENFERMERÍA}

En torno al rol político, Hannah Arendt promueve la libertad individual, rechazando las ideas totalitarias y los fundamentalismos como sistemas que sepultan y despersonalizan al ser humano. En el contexto público-político de la «polis» todos los asuntos se resuelven en un contexto relacional, a través del diálogo y la persuasión entre iguales. En este ámbito las personas despliegan su capacidad de acción y diálogo, siendo espontáneos, innovadores, en definitiva, libres ${ }^{(18)}$. 
Desde una perspectiva comprensiva actualizada del rol sociopolítico del profesional de enfermería, entendiendo a la política como una actividad orientada en forma ideológica a la toma de decisiones de un grupo comprometido como ciudadanas y ciudadanos, en pos de alcanzar objetivos comunes, dentro del contexto social al cual pertenecen.

En nuestro caso, podríamos afirmar que se requiere de un empoderamiento político y participativo al interior de los colectivos gremiales, como una forma de fortalecer el discurso y llegar a acciones concretas. Desde la perspectiva social, se debe ejercer poder para resolver los requerimientos sanitarios de las comunidades, a través del conocimiento de los espacios donde se toman las decisiones políticas y encontrar la manera de participar en ellos e influir en los procesos decisionales ${ }^{(19-21)}$. Este empoderamiento sociopolítico debe considerar, el compromiso con las problemáticas antiguas y emergentes de la profesión, con una mirada de futuro, como componentes de una nueva enfermería.

\section{CONCLUYENDO}

Si el compromiso es solidariedad, acercamiento a los problemas de las personas de manera sistemática, en un «estar ahí, siempre», de manera auténtica ${ }^{(22)}$. Ese estar ahí, señala la ruta a seguir como profesionales sensibles al contexto social y político de nuestro país, asumiendo un rol que les hace responsables de identificar los patrones de los determinantes sociales y su impacto al interior de las familias y comunidades, producto de la actual crisis sociosanitaria, para apoyarles en sus decisiones en pos de su bienestar.

En este sentido, la invitación es a analizar la realidad con ojos críticos y hacer propuestas para mejorar las condiciones de salud de la comunidad. Poniendo atención a las migraciones humanitarias, que incorporan otras lenguas, otros patrones de salud y de crianza; a la reaparición de enfermedades infecciosas que estaban en franca retirada; al cuidado del medio ambiente; a la violencia de género; a la violencia infanto-juvenil; a los adultos mayores en condiciones de abandono; a las personas en condición de calle; a la discriminación en todos los escenarios en que se presente; a la contaminación ambiental; a la ampliación de nuevas enfermedades a las Garantías Explícitas en Salud, así como estas, se les invita a pensar en un extenso etcétera....

Agregaremos, el compromiso con nuestras luchas gremiales, enfrentados a problemas aún no resueltos, como la exclusividad formativa universitaria; el cuidado de enfermería en unidades de neonatología; los estándares comunes de la calidad formativa; el cumplimiento de los indicadores de ratio enfermeras/pacientes en el sistema de salud público y privado; la creciente oferta formativa y la precarización de la empleabilidad, entre otros.

En la formación se propone una nueva enfermería que posicione a los noveles profesionales como líderes expertos, entendidos y poseedores de poder para tomar decisiones, en los contextos en que desempeñe sus funciones, asistencial, docente, investigativa y en los niveles de la gestión, macro, meso y micro.

La nueva enfermería propuesta, requiere incorporar a la innovación, como una función incluida en el rol, considerando la productividad de propias investigaciones, que generen nuevas formas de cumplir con las áreas derivadas del rol. Como condición para que 
ello ocurra, es que el colectivo valide las innovaciones propuestas, en un acto solidario de reconocimiento interpares.

\section{REFERENCIAS BIBLIOGRÁFICAS}

1. Biblioteca del Congreso Nacional de Chile. Ley de Autoridad Sanitaria 19.536. Artículo 113. Promulgada 28, noviembre, 1997. URL http://bcn.cl/1uvqh

2. Reglamento Orgánico del Ministerio de Salud. Norma General Administrativa No 19. Gestión del cuidado de enfermería para la atención cerrada. Resolución exenta 1127.14 diciembre, $2007 . \quad$ Disponible en: https://enfermeriachl.files.wordpress.com/2018/11/resolucion-nc2b0-1127-normanc2b0-19.pdf@

3. González-Jurado, M. El compromiso de la Enfermería con la sociedad. 2009; Rev. Administración Sanit. Siglo XXI; 7(2):183-356. Disponible en: http://www.elsevier.es/es-revista-revista-administracion-sanitaria-siglo-xxi-261articulo-el-compromiso-enfermeria-con-sociedad-13139734

4. Consejo Internacional de Enfermeras. Declaración de Posición. Disponible en: https://www.icn.ch/es/politica-de-enfermeria/declaracion-de-posicion

5. Landman, C., Arriola, Y., Chacón, A., Giorgis De, A., Esparza, C., E. Herrera, E.F, P. Pizarro, P., Ribera, S., Rojas, D., Verdejo, V. y J. Molina'J. Transitando hacia el ejercicio de autonomía y liderazgo: ... «para el posicionamiento hay que luchar» Enferm. univ [revista en la Internet]. 2019 Jun [citado 2021 Abr 05]; 16(2): 157-170. Disponible en: http://www.scielo.org.mx/scielo.php?script=sci_arttext\&pid=S166570632019000200157\&lng=es. https://doi.org/10.22201/eneo.23958421e.2019.2.644.

6. Ley $\mathrm{N}^{\mathrm{0}} 20.584$ Regula los derechos y deberes que tienen las personas en relación con acciones vinculadas a su atención en salud. Biblioteca Congreso Nacional de Chile. Disponible en: https://www.leychile.cl/Navegar?idNorma=1039348

7. Scully, N. Leadership in nursing: The importance of recognizing inherent values and attributes to secure a positive future for the profession. ACN. 2015; 22 (4):439-44. https://doi.org/10.1016/j.colegn.2014.09.004

8. Yáñez, R. y Valenzuela, S. Conductas crítica para experimentar confianza en el liderazgo en enfermería en un hospital de alta complejidad. Aquichan.2013; 13(2):186-96. https://doi.org/10.5294/aqui.2013.13.2.4

9. Tapp, D., Stansfield, K. y Stewart, J. La autonomía en la práctica de enfermería. Aquichán. 2005; 5(1):114-27. Disponible en: http://www.scielo.org.co/scielo.php?pid=S1657$59972005000100012 \&$ script=sci_abstract\&tlng=es

10. Quezada, C., Illesca, M. y Cabezas, M. Percepción del liderazgo en las(os) enfermeras(os) de un hospital del sur de Chile. Cienc enferm. 2014; 20(2):41-51. http://dx.doi.org/10.4067/S0717-95532014000200005 
11. Garlet, E., de Lima L., Dias da Silva, $M$ et al. The resignification of management processes in care procedures in nursing. OBJN. 2006;5(3). http://www.objnursing.uff.br/index.php/nursing/article/view/613

12. Drucker, P. El ejecutivo eficaz. Argentina: Ed. de bolsillo; 2014.

13. Guerrero, S. y Cid, P. Una reflexión sobre la autonomía y el liderazgo en enfermería. Aquichan. 2015; 15(1):129-40. http://dx.doi.org/10.5294/aqui.2015.15.1.12

14. Letelier, M. y Velandia, AL. Profesionalismo en enfermería, el hábito de la excelencia del cuidado. Av.enferm.-Col-2010;28(2):145-158 https://revistas.unal.edu.co/index.php/avenferm/article/view/21391

15. Souza e Souza, L., Ferreira, A., Nunes de Aguiar R, et al. El liderazgo en la visión de enfermeros líderes. Enferm Global.2013;12(30):268-80. http://dx.doi.org/10.6018/eglobal.12.2.154801

16. Luengo, C. y Paravic, T. Autonomía Profesional: factor clave para el ejercicio de la Enfermería Basada en la Evidencia. Index Enferm.2016;25(1-2):42-46. http://scielo.isciii.es/scielo.php?script=sci_arttext\&pid=S113212962016000100010\&ln $\mathrm{g}=\mathrm{es}$

17. Heierle, C. La imagen de la enfermera a través de los medios de comunicación de masas: La prensa escrita. Index Enferm [Internet]. 2009 Jun [citado 2021 Jun 25]; 18(2): 9598. Disponible en: http://scielo.isciii.es/scielo.php?script=sci_arttext\&pid=S1132$12962009000200005 \& \operatorname{lng}=\mathrm{es}$.

18. Arendt, A. ¿Qué es política? recopilación realizada por Úrsula Ludz de conferencias y artículos de la autora. Traducido por Rosa Sala Carbó. Ediciones Paidós: Barcelona, 1997.

19. Rutz Porto Adrize, Buss Thofehrn Maira. El empoderamiento político de los enfermeros en la práctica hospitalaria. Index Enferm [Internet]. 2015 Jun [citado 2019 Mar 22] ; 24( 1-2 20-24. $)$ Disponible http://scielo.isciii.es/scielo.php?script=sci_arttext\&pid=S113212962015000100005\&lng=es. http://dx.doi.org/10.4321/S1132-12962015000100005.

20. Megías-Lizancos F. El compromiso social de las enfermeras. Rev. Portuguesa de Enferm. de Saúde Mental [Internet]. 2017 Jun [citado 2019 Mar 25]; (17):6-7. Disponível em: http://www.scielo.mec.pt/scielo.php?script=sci_arttext\&pid=S164721602017000100001\&lng=pt. http://dx.doi.org/10.19131/rpesm.0177.

21. Gutiérrez-Valverde, J. y Nuñez-Rocha, G. La participación de enfermería en la formulación de políticas de salud. Rev Enferm Inst Mex Seguro Soc 2009; 17 (1): 5960. Disponible en: http://www.medigraphic.com/pdfs/enfermeriaimss/eim2009/eim091j.pdf

22. Heidegger, Martin. Ser y Tiempo. Trad. Jorge E. Rivera. Madrid: Trotta, 2012. 